\title{
Eficácia do Carfentrazone-Ethyl no Controle de Plantas Aquáticas latifoliadas em CaiXas-D'ÁGua ${ }^{1}$
}

\author{
Efficiency of Carfentrazone-Ethyl in Controlling Large-Leafed Aquatic Plants Using Boxes of \\ Water
}

\author{
CARVALHO, F.T. ${ }^{2}$, VELINI, E.D. ${ }^{2}$, NEGRISOLI, E. ${ }^{3}$ e ROSSI, C.V.S. ${ }^{4}$
}

\begin{abstract}
RESUMO - Atualmente, alguns herbicidas estão sendo desenvolvidos para o controle de plantas daninhas aquáticas. O objetivo deste trabalho foi avaliar a eficácia do carfentrazoneethyl em ambiente aquático para o controle pós-emergente de aguapé, alface-d'água e salvinia. O trabalho foi desenvolvido em caixas-d'água, no período de julho a setembro de 2004, no NUPAM - FCA/UNESP, em Botucatu. O delineamento experimental adotado foi o de blocos ao acaso, com sete tratamentos e quatro repetições, sendo as unidades experimentais constituídas pelas caixasd'água. Os tratamentos foram os seguintes: testemunha sem herbicida; Aurora $400 \mathrm{CE}\left(75,150\right.$ e $\left.300 \mathrm{~mL} \mathrm{ha}^{-1}\right)$; Roundup $\left(3,0 \mathrm{~L} \mathrm{ha}^{-1}\right)$, Aurora $400 \mathrm{CE}+$ Roundup $\left(75 \mathrm{~mL}+3,0 \mathrm{~L} \mathrm{ha}^{-1}\right)$ e Aurora $400 \mathrm{CE}+$ Arsenal N.A. $(75 \mathrm{~mL}+$ 2,0 L ha $\left.{ }^{-1}\right)$. Observou-se que o tratamento Aurora $400 \mathrm{CE}\left(300 \mathrm{~mL} \mathrm{ha}^{-1}\right)$ é altamente eficaz no controle de alface-d'água (Pistia stratiotes); o tratamento Roundup $\left(3,0 \mathrm{~L} \mathrm{ha}^{-1}\right)$ é altamente eficaz no controle de aguapé (Eichhornia crassipes); o tratamento Aurora $400 \mathrm{CE}+$ Roundup $\left(75 \mathrm{~mL}+3,0 \mathrm{~L} \mathrm{ha}^{-1}\right)$ é eficaz no controle de aguapé (E. crassipes), alface-d'água ( $P$. stratiotes) e salvínia (Salvinia auriculata); e o tratamento Aurora $400 \mathrm{CE}+\operatorname{Arsenal}\left(75 \mathrm{~mL}+2,0 \mathrm{~L} \mathrm{ha}^{-1}\right)$ é eficaz no controle de aguapé (E. crassipes) e alface-d'água ( $P$. stratiotes). A mistura Aurora $400 \mathrm{CE}+$ Roundup $\left(75 \mathrm{~mL}+3,0 \mathrm{~L} \mathrm{ha}^{-1}\right)$ apresentou-se viável e foi o único tratamento eficaz no controle das três espécies estudadas.
\end{abstract}

Palavras-chave: planta daninha, herbicida, Salvinia auriculata, Eichhornia crassipes, Pistia stratiotes.

\begin{abstract}
Some herbicides are being currently developed for the control of aquatic weeds. The objective of this work was to evaluate the efficiency of Aurora $400 \mathrm{CE}$ in aquatic environment for the post-emergence control of Eichhornia crassipes, Pistia stratiotes and Salvinia auriculata. The experiment was developed in boxes of water, from 22/07 to 20/09/2004, at NUPAM-FCA/ UNESP, Botucatu, SP, Brazil. The experimental design was in randomized blocks with seven treatments and four repetitions, with the units consisting of boxes of water. The following treatments were used: control without herbicide; Aurora $400 \mathrm{CE}$ (75, 150 and $300 \mathrm{~mL} \mathrm{ha}^{-1}$ ); Roundup (3, O L ha $\left.\mathrm{h}^{-1}\right)$; Aurora $400 \mathrm{CE}+$ Roundup (75 $\left.\mathrm{mL}+3,0 \mathrm{~L} \mathrm{ha}^{-1}\right)$ and Aurora $400 \mathrm{CE}+$ Arsenal N.A. $\left(75 \mathrm{~mL}^{2} 2.0 \mathrm{~L} \mathrm{ha}^{-1}\right)$. It was observed that the treatment with Aurora $400 \mathrm{CE}\left(300 \mathrm{~mL} \mathrm{ha}^{-1}\right)$ is highly efficient in the control of Pistia stratiotes; Roundup $\left(3,0 \mathrm{~L} \mathrm{ha}^{-1}\right)$ is highly efficient in the control of Eichhornia crassipes; Aurora $400 \mathrm{CE}+$ Roundup $\left(75 \mathrm{~mL}+3.0 \mathrm{~L} \mathrm{ha}^{-1}\right)$ is efficient in the control of $\boldsymbol{E}$. crassipes, $\boldsymbol{P}$. stratiotes and Salvinia auriculata and Aurora $400 \mathrm{CE}+$ Arsenal $(75 \mathrm{~mL}$ $+2.0 \mathrm{~L} \mathrm{ha}^{-1}$ ) are efficient in the control of $\boldsymbol{E}$. crassipes and $\boldsymbol{P}$. stratiotes. The mixture Aurora $400 \mathrm{CE}+$ Roundup $\left(75 \mathrm{~mL}+3.0 \mathrm{~L} \mathrm{ha}^{-1}\right)$ was viable and the only efficient treatment in the control of the three studied species.
\end{abstract}

Key words: weed, herbicide, Salvinia auriculata, Eichhornia crassipes, Pistia stratiotes.

Recebido para publicação em 12.2.2005 e na forma revisada em 8.4.2005.

2 Prof. Dr., Dep. de Biologia e Zootecnia, FEIS/UNESP, Av. Brasil, no 56, Centro, 15385-000 Ilha Solteira-SP, $<$ ftadeu@bio.feis.unesp.br>; ${ }^{3}$ Prof. Dr., Departamento de Agricultura, FCA/UNESP, Botucatu-SP. ${ }^{4}$ Eng.-Agr., M.S., Doutorando em Agricultura, FCA/UNESP, Botucatu-SP. 


\section{INTRODUÇÃO}

As plantas aquáticas são estudadas como um efeito do desequilíbrio causado pela poluição e, ou, represamento dos rios. A quantidade excessiva de plantas, conseqüente desse desequilíbrio, dificulta a navegação e a produção de energia elétrica.

A ocorrência de plantas aquáticas em reservatórios de hidrelétricas é um problema de importância crescente no Brasil. Algumas usinas tiveram sua eficiência comprometida pelas elevadas infestações de plantas emersas e imersas, como a hidrelétrica de Jupiá, que, em alguns meses do ano, tem seu funcionamento comprometido devido ao entupimento das grades de proteção das turbinas por grandes massas de plantas aquáticas. Em outras companhias, o problema com plantas aquáticas tem sido constante. Na Light, por exemplo, o custo anual com controle de macrófitas é da ordem de $\mathrm{R} \$$ 3.000.000,00 (Velini, 1998).

O conceito de plantas indesejáveis, no tempo e espaço, utilizado para caracterizá-las como daninhas, se enquadra perfeitamente nos casos das macrófitas quando seu crescimento acentuado causa dificuldades para a utilização dos ecossistemas aquáticos. Quando esse nivel de dano é atingido, passa a ser necessária a aplicação de métodos de manejo que minimizem suas populações.

As plantas daninhas aquáticas flutuantes, como aguapé (Eichhornia crassipes), alfaced'água (Pistia stratiotes) e salvinia (Salvinia auriculata), são as que causam os mais sérios e difundidos problemas em nível mundial. Atualmente, alguns herbicidas estão sendo desenvolvidos para o controle dessas plantas. Esses produtos devem ser amplamente estudados, já que se trata de um ecossistema diferenciado.

A espécie E. crassipes é uma das mais disseminadas do mundo; trata-se de uma planta aquática, perene, flutuante livre, nativa da Amazônia e que se propaga basicamente por brotações de talos (Swarbrick, 1981; Kissmann, 1997; Lorenzi, 2000).

As plantas de $P$. stratiotes e $S$. auriculata são espécies cosmopolitas com ampla distribuição por todo o planeta (University of
Florida, 2004). P. stratiotes é uma planta aquática perene, flutuante livre, nativa da América Tropical e que se propaga por estolões. Já S. auriculata é uma planta aquática, flutuante livre, nativa da América do Sul e que se propaga vegetativamente por brotações (Kissmann, 1997; Lorenzi, 2000).

O herbicida carfentrazone possui excelente eficácia de controle das plantas latifoliadas e um ótimo perfil toxicológico, o que o credencia com grande potencial para o uso em ambientes aquáticos. Dessa forma, o presente trabalho foi desenvolvido com o objetivo de avaliar a eficácia do Aurora $400 \mathrm{CE}$ em ambiente aquático para o controle pós-emergente das macrófitas aquáticas: aguapé, alface-d'água e salvinia.

\section{MATERIAL E MÉTODOS}

O experimento foi desenvolvido no período de 22 de julho a 20 de setembro de 2004, no Núcleo de Pesquisas Avançadas em Matologia (NUPAM) da FCA/UNESP- Botucatu. Realizouse em caixas-d'água e as espécies utilizadas foram aguapé, alface-d'água e salvínia, que foram mantidas no ambiente utilizando uma quantidade uniforme de plantas para cada espécie, suficiente para cobrir toda a superficie da água. Foi mantido o nível de água nas caixas por meio de um sistema de bóias. Os tratamentos utilizados no experimento estão apresentados na Tabela 1.

Utilizaram-se os herbicidas descritos a seguir.

Aurora 400 CE (nome comercial): carfentrazone-ethyl, etil-2-cloro-3-[2-cloro-4fluoro-5-[4-(difluoro-4,5-diidro-3-metil-5-oxo1H-1,2,4-triazol-1-il)fenil] propanoato, pertencente ao grupo das aril triazolinonas, apresentado como concentrado emulsionável (CE) contendo $400 \mathrm{~g} \mathrm{~L}^{-1}$ de ingrediente ativo (i.a.), sendo um herbicida latifolicida e com classe toxicológica II, comercializado pela FMC do Brasil Indústria e Comércio S.A.

Roundup (nome comercial): glyphosate, sal de isopropilamina de $\mathrm{N}$-(fosfonometil) glicina, pertencente ao grupo dos derivados da glicina, apresentado como concentrado solúvel (CS) contendo $480 \mathrm{~g} \mathrm{~L}^{-1}$ de i.a., sendo um herbicida sistêmico não-seletivo e com classe 
Tabela 1 - Tratamentos utilizados no experimento. FCA/ UNESP - Botucatu/SP, 2004

\begin{tabular}{|c|c|c|}
\hline \multirow{2}{*}{ Tratamento } & \multicolumn{2}{|c|}{ Dose dos Herbicidas } \\
\hline & Produto comercial por hectare & Ingrediente ativo $\left(\mathrm{g} \mathrm{ha}^{-1}\right)$ \\
\hline 1- Testemunha sem herbicida & - & - \\
\hline 2- Aurora $400 \mathrm{CE}$ & $75 \mathrm{~mL}$ & 30,0 \\
\hline 3- Aurora $400 \mathrm{CE}$ & $150 \mathrm{~mL}$ & 60,0 \\
\hline 4- Aurora $400 \mathrm{CE}$ & $300 \mathrm{~mL}$ & 120,0 \\
\hline 5- Roundup & $3,0 \mathrm{~L}$ & $1.440,0$ \\
\hline 6- Aurora $400 \mathrm{CE}+$ Roundup & $75 \mathrm{~mL}+3,0 \mathrm{~L}$ & $30,0+1.440,0$ \\
\hline 7- Aurora $400 \mathrm{CE}+$ Arsenal N.A. & $75 \mathrm{~mL}+2,0 \mathrm{~L}$ & $30,0+532,6$ \\
\hline
\end{tabular}

toxicológica IV, comercializado pela Monsanto do Brasil Ltda.

Arsenal N.A. (nome comercial): imazapyr, sal de amônia do ácido nicotínico 2-(4-isopropil)4-metil-5oxo-imidazolina, pertencente ao grupo das imidazolinonas, apresentado como concentrado solúvel (CS) contendo $250 \mathrm{~g} \mathrm{~L}^{-1} \mathrm{de}$ i.a., sendo um herbicida sistêmico e com classe toxicológica III, comercializado pela Basf S.A.

O delineamento experimental adotado foi de blocos ao acaso, com sete tratamentos e quatro repetições. As unidades experimentais (parcelas) foram constituídas pelas caixasd'água de 60 × 60 × $60 \mathrm{~cm}$, com 120 litros de água e uma camada de solo de 20 litros. As plantas de aguapé (E. crassipes) foram colocadas em caixas individuais e as de alfaced'água ( $P$. stratiotes) e salvínia ( $S$. auriculata) nas mesmas caixas, porém divididas por meio de uma tela.

As aplicações dos herbicidas foram feitas com um pulverizador costal a pressão constante de $\mathrm{CO}_{2}$ a $45 \mathrm{lb}$ pol$^{-2}$, provido de tanque com capacidade de dois litros (garrafas descartáveis) e barra equipada com dois bicos do tipo leque (Teejet $110.02 \mathrm{XR}$ ), espaçados de $0,5 \mathrm{~m}$, sendo o volume de calda gasto equivalente a $200 \mathrm{~L} \mathrm{ha}^{-1}$.

Realizaram-se as aplicações no dia 22.7.2004, entre $16 \mathrm{~h}$ e $16 \mathrm{~h} 30$. As condições climáticas no momento apontavam para uma temperatura de $20^{\circ} \mathrm{C}$, umidade relativa do ar de $67 \%$ e ausência de vento. Apesar disso, os tratamentos vizinhos foram protegidos com lona plástica, para evitar qualquer tipo de deriva.
As avaliações de eficácia dos herbicidas no controle das plantas aquáticas foram feitas aos $6,14,20,26,36,43,50$ e 60 dias após a aplicação (DAA), por meio de uma escala visual, em que $0 \%$ corresponde a nenhum controle e $100 \%$ a controle total das plantas. Considerou-se eficaz o controle igual ou superior a $80 \%$, sendo os dados analisados estatisticamente através do teste de Tukey.

\section{RESULTADOS}

Observaram-se diferenças nos tratamentos em relação à eficácia de controle sobre as diferentes espécies testadas. A partir da escala visual, os resultados estão descritos a seguir para aguapé, alface-d'água e salvínia, nas Tabelas 2, 3 e 4, respectivamente, tratando-se separadamente cada uma.

\section{Aguapé (Eichhornia crassipes)}

Observa-se que o controle sobre aguapé proporcionado pelo Aurora $400 \mathrm{CE}(75,150$ e $300 \mathrm{~mL} \mathrm{ha}^{-1}$ ) aplicado isoladamente foi pouco eficaz. Apesar de o tratamento com $300 \mathrm{~mL}^{\text {ha }}{ }^{-1}$ ter atingido $82,5 \%$ no período de 36 aos 50 DAA, houve recuperação das plantas e o controle tornou-se ineficaz aos 60 DAA (Tabela 2).

No caso do Roundup (3,0 L ha-1), foi altamente eficaz no controle da planta, proporcionando niveis totais $(100 \%)$ aos 60 DAA. O tratamento Aurora $400 \mathrm{CE}+$ Roundup $(75 \mathrm{~mL}$ + 3,0 $\mathrm{L} \mathrm{ha}^{-1}$ ) também apresentou a mesma eficácia, proporcionando niveis médios de $98 \%$ aos 60 DAA (Tabela 2).

Em relação ao tratamento Aurora $400 \mathrm{CE}$ + Arsenal $\left(75 \mathrm{~mL}+2,0 \mathrm{~L} \mathrm{ha}^{-1}\right)$, a eficácia foi 
Tabela 2 - Porcentagem de controle de Eichhornia crassipes em diferentes períodos, após a aplicação dos herbicidas. FCA/UNESP - Botucatu-SP, 2004

\begin{tabular}{|c|c|c|c|c|c|c|c|c|}
\hline \multirow{2}{*}{ Tratamento } & \multicolumn{8}{|c|}{ Dias após a aplicação } \\
\hline & 6 & 14 & 20 & 26 & 36 & 43 & 50 & 60 \\
\hline 1-Testemunha sem herbicida & 0,0 & 0,0 & 0,0 & 0,0 & 0,0 & 0,0 & 0,0 & 0,0 \\
\hline 2-Aurora $400 \mathrm{CE}\left(75 \mathrm{~mL} \mathrm{ha}^{-1}\right)$ & $42,5 \mathrm{~b}$ & $70,8 \mathrm{~b}$ & $72,8 \mathrm{~b}$ & " $76,3 \mathrm{~b}$ & $51,8 \mathrm{~d}$ & $46,3 \mathrm{~d}$ & $46,3 \mathrm{e}$ & $20,0 \mathrm{~d}$ \\
\hline 3-Aurora $400 \mathrm{CE}\left(150 \mathrm{~mL} \mathrm{ha}^{-1}\right)$ & $42,5 \mathrm{~b}$ & $68,0 \mathrm{~b}$ & $68,0 \mathrm{~b}$ & $75,3 \mathrm{~b}$ & $70,0 \mathrm{c}$ & $70,0 \mathrm{c}$ & $63,8 \mathrm{~d}$ & $55,0 \mathrm{c}$ \\
\hline 4-Aurora $400 \mathrm{CE}\left(300 \mathrm{~mL} \mathrm{ha}^{-1}\right)$ & $42,5 \mathrm{~b}$ & $68,8 \mathrm{~b}$ & $72,5 \mathrm{~b}$ & $80,0 \mathrm{~b}$ & $82,5 \mathrm{~b}$ & $82,5 \mathrm{~b}$ & $82,5 \mathrm{c}$ & $68,8 \mathrm{~b}$ \\
\hline 5-Roundup $\left(3,0 \mathrm{~L} \mathrm{ha}^{-1}\right)$ & $39,5 \mathrm{~b}$ & $88,0 \mathrm{a}$ & $92,3 \mathrm{a}$ & $94,8 \mathrm{a}$ & $96,5 \mathrm{a}$ & $98,0 \mathrm{a}$ & $98,5 \mathrm{a}$ & $100,0 \mathrm{a}$ \\
\hline $\begin{array}{l}\text { 6-Aurora } 400 \mathrm{CE}+\text { Roundup } \\
\left(75 \mathrm{~mL}+3,0 \mathrm{~L} \mathrm{ha}^{-1}\right)\end{array}$ & $61,3 \mathrm{a}$ & $90,0 \mathrm{a}$ & $93,8 \mathrm{a}$ & $93,8 \mathrm{a}$ & 94,8 a & $94,8 \mathrm{a}$ & $96,8 \mathrm{ab}$ & $98,0 \mathrm{a}$ \\
\hline $\begin{array}{l}\text { 7-Aurora } 400 \mathrm{CE}+\text { Arsenal } \\
\left(75 \mathrm{~mL}+2,0 \mathrm{~L} \mathrm{ha}^{-1}\right)\end{array}$ & $35,0 \mathrm{~b}$ & $52,5 \mathrm{c}$ & $55,5 \mathrm{c}$ & $56,0 \mathrm{c}$ & $64,5 \mathrm{c}$ & $73,8 \mathrm{c}$ & $92,0 \mathrm{~b}$ & $95,5 \mathrm{a}$ \\
\hline F. tratamento & $9,1 * *$ & $49,8^{* *}$ & $48,5 * *$ & $63,5^{* *}$ & $118,4^{* *}$ & $148,3 * *$ & $220,4 * *$ & $124,9 * *$ \\
\hline CV $(\%)$ & 13,62 & 5,44 & 5,59 & 4,51 & 4,24 & 4,01 & 3,51 & 7,78 \\
\hline DMS $(5 \%)$ & 13,74 & 9,14 & 9,74 & 8,23 & 7,48 & 7,15 & 6,46 & 13,03 \\
\hline
\end{tabular}

** significativo a $1 \%$ de probabilidade. Médias seguidas de mesma letra, na coluna, não diferem estatisticamente entre si pelo teste $\mathrm{t}(\mathrm{P}>0,05)$.

Tabela 3 - Porcentagem de controle de Pistia stratiotes em diferentes períodos, após a aplicação dos herbicidas. FCA/UNESP - Botucatu-SP, 2004

\begin{tabular}{|c|c|c|c|c|c|c|c|c|}
\hline \multirow{2}{*}{ Tratamento } & \multicolumn{8}{|c|}{ Dias após a aplicação } \\
\hline & 6 & 14 & 20 & 26 & 36 & 43 & 50 & 60 \\
\hline 1-Testemunha sem herbicida & 0,0 & 0,0 & 0,0 & 0,0 & 0,0 & 0,0 & 0,0 & 0,0 \\
\hline 2-Aurora $400 \mathrm{CE}\left(75 \mathrm{~mL} \mathrm{ha}{ }^{-1}\right)$ & $33,8 \mathrm{~b}$ & $75,3 \mathrm{c}$ & $76,0 \mathrm{~b}$ & $53,8 \mathrm{bc}$ & $30,0 \mathrm{~d}$ & $12,5 \mathrm{c}$ & $6,3 \mathrm{c}$ & $0,0 \mathrm{c}$ \\
\hline 3-Aurora $400 \mathrm{CE}\left(150 \mathrm{~mL} \mathrm{ha}^{-1}\right)$ & $40,0 \mathrm{~b}$ & $77,0 \mathrm{bc}$ & $79,3 \mathrm{~b}$ & $62,5 \mathrm{~b}$ & $30,0 \mathrm{~d}$ & $15,0 \mathrm{c}$ & $5,0 \mathrm{c}$ & $2,5 \mathrm{c}$ \\
\hline 4-Aurora $400 \mathrm{CE}\left(300 \mathrm{~mL} \mathrm{ha}^{-1}\right)$ & $85,8 \mathrm{a}$ & $90,0 \mathrm{a}$ & $90,0 \mathrm{a}$ & $95,8 \mathrm{a}$ & $96,3 \mathrm{a}$ & $96,3 \mathrm{a}$ & $96,3 \mathrm{a}$ & $96,5 \mathrm{a}$ \\
\hline 5-Roundup $\left(3,0 \mathrm{~L} \mathrm{ha}^{-1}\right)$ & $8,8 \mathrm{c}$ & $50,0 \mathrm{~d}$ & $51,8 \mathrm{c}$ & $50,5 \mathrm{c}$ & $53,8 \mathrm{c}$ & $46,3 \mathrm{~b}$ & $28,8 \mathrm{~b}$ & $28,8 \mathrm{~b}$ \\
\hline $\begin{array}{l}\text { 6-Aurora } 400 \mathrm{CE}+\text { Roundup } \\
\left(75 \mathrm{~mL}+3,0 \mathrm{~L} \mathrm{ha}^{-1}\right)\end{array}$ & $33,8 \mathrm{~b}$ & $81,8 \mathrm{abc}$ & $89,5 \mathrm{a}$ & $88,0 \mathrm{a}$ & $80,3 \mathrm{~b}$ & $80,0 \mathrm{a}$ & $80,0 \mathrm{~b}$ & 80,0 a \\
\hline $\begin{array}{l}\text { 7-Aurora } 400 \mathrm{CE}+\text { Arsenal }^{-1} \\
\left(75 \mathrm{~mL}+2,0 \mathrm{~L} \mathrm{ha}^{-1}\right)\end{array}$ & $40,0 \mathrm{~b}$ & $87,5 \mathrm{ab}$ & $87,5 \mathrm{a}$ & 89,0 a & $87,0 \mathrm{ab}$ & $87,5 \mathrm{a}$ & 86,3 a & $86,3 \mathrm{a}$ \\
\hline F. tratamento & $69,7 * *$ & $38,5 * *$ & $83,8^{* *}$ & $107,8 * *$ & $73,0 * *$ & $99,5 * *$ & $122,8 * *$ & $134,6^{* *}$ \\
\hline $\mathrm{CV}(\%)$ & 14,90 & 6,03 & 4,02 & 5,24 & 10,85 & 13,20 & 14,97 & 15,40 \\
\hline DMS $(5 \%)$ & 13,82 & 10,66 & 7,30 & 8,83 & 15,68 & 17,07 & 17,36 & 17,36 \\
\hline
\end{tabular}

** significativo a $1 \%$ de probabilidade. Médias seguidas de mesma letra, na coluna, não diferem estatisticamente entre si pelo teste $t(P>0,05)$.

alta sobre o aguapé, proporcionando níveis médios de controle de $95,5 \%$ aos 60 DAA; entretanto, ressalta-se que, entre os tratamentos eficazes, este apresentou o controle mais lento (Tabela 2).

Analisando os dados de evolução das eficácias de controle de aguapé, destacaram-se os tratamentos 5 (Roundup, com 3,0 L ha ${ }^{-1}$ ) e 6 (Aurora $400 \mathrm{CE}+$ Roundup, com $75 \mathrm{~mL}$ + 3,0 L ha $\left.{ }^{-1}\right)$. A semelhança na eficácia desses tratamentos para o aguapé significa que não houve efeito antagônico para o controle. Apesar de não ter sido necessária ao Roundup a mistura com Aurora $400 \mathrm{CE}$, no caso do aguapé, o resultado foi importante, demonstrando que a mistura é possivel, já que o Roundup isolado não é eficaz para as demais espécies aquáticas.

\section{Alface-d'água (Pistia stratiotes)}

Na Tabela 3, observa-se que o herbicida Aurora $400 \mathrm{CE}\left(300 \mathrm{~mL} \mathrm{ha}^{-1}\right)$, aplicado isoladamente, proporcionou eficácia no controle 
Tabela 4 - Porcentagem de controle de Salvinia auriculata em diferentes períodos, após a aplicação dos herbicidas. FCA/UNESP - Botucatu-SP, 2004

\begin{tabular}{|c|c|c|c|c|c|c|c|c|}
\hline \multirow{2}{*}{ Tratamento } & \multicolumn{8}{|c|}{ Dias após a aplicação } \\
\hline & 6 & 14 & 20 & 26 & 36 & 43 & 50 & 60 \\
\hline 1-Testemunha sem herbicida & 0,0 & 0,0 & 0,0 & 0,0 & 0,0 & 0,0 & 0,0 & 0,0 \\
\hline 2-Aurora $400 \mathrm{CE}\left(75 \mathrm{~mL} \mathrm{ha}^{-1}\right)$ & $28,8 \mathrm{c}$ & $67,5 \mathrm{c}$ & $71,3 \mathrm{c}$ & $63,0 \mathrm{~b}$ & $36,5 \mathrm{de}$ & $17,5 \mathrm{c}$ & $13,8 \mathrm{c}$ & $0,0 \mathrm{~b}$ \\
\hline 3-Aurora $400 \mathrm{CE}\left(150 \mathrm{~mL} \mathrm{ha}^{-1}\right)$ & $47,0 \mathrm{~b}$ & $68,8 \mathrm{c}$ & $73,0 \mathrm{bc}$ & $56,3 \mathrm{~b}$ & $30,0 \mathrm{e}$ & $23,8 \mathrm{c}$ & $9,5 \mathrm{c}$ & $2,5 \mathrm{~b}$ \\
\hline 4-Aurora $400 \mathrm{CE}\left(300 \mathrm{~mL} \mathrm{ha}^{-1}\right)$ & $73,8 \mathrm{a}$ & $85,0 \mathrm{ab}$ & $81,3 \mathrm{ab}$ & $83,3 \mathrm{a}$ & $65,5 \mathrm{bc}$ & $65,5 \mathrm{a}$ & $65,5 \mathrm{ab}$ & $10,0 \mathrm{~b}$ \\
\hline 5-Roundup $\left(3,0 \mathrm{~L} \mathrm{ha}^{-1}\right)$ & $7,5 \mathrm{~d}$ & $37,5 \mathrm{~d}$ & $52,5 \mathrm{~d}$ & $50,8 \mathrm{~b}$ & $50,8 \mathrm{~cd}$ & $59,5 \mathrm{ab}$ & $59,5 \mathrm{~b}$ & $59,5 \mathrm{a}$ \\
\hline $\begin{array}{l}\text { 6-Aurora } 400 \mathrm{CE}+\text { Roundup } \\
\left(75 \mathrm{~mL}+3,0 \mathrm{~L} \mathrm{ha}^{-1}\right)\end{array}$ & $28,8 \mathrm{c}$ & 88,0 a & 88,0 a & 87,3 a & $83,8 \mathrm{a}$ & 83,3 a & $82,5 \mathrm{a}$ & $82,5 \mathrm{a}$ \\
\hline $\begin{array}{l}\text { 7-Aurora } 400 \mathrm{CE}+\text { Arsenal } \\
\left(75 \mathrm{~mL}+2,0 \mathrm{~L} \mathrm{ha}^{-1}\right)\end{array}$ & $45,0 \mathrm{~b}$ & $74,8 \mathrm{bc}$ & $90,3 \mathrm{a}$ & 87,0 a & $76,8 \mathrm{ab}$ & $36,3 \mathrm{bc}$ & $16,3 \mathrm{c}$ & $13,8 \mathrm{~b}$ \\
\hline F. tratamento & $113,7 * *$ & $63,30 * *$ & $45,56^{* *}$ & $26,1 * *$ & $30,2 * *$ & $17,9 * *$ & $44,8 * *$ & $36,7^{* *}$ \\
\hline CV $(\%)$ & 10,92 & 6,47 & 5,40 & 9,07 & 13,85 & 25,64 & 22,99 & 40,54 \\
\hline DMS $(5 \%)$ & 9,66 & 10,46 & 9,44 & 14,86 & 18,22 & 28,08 & 21,77 & 26,15 \\
\hline
\end{tabular}

** significativo a $1 \%$ de probabilidade. Médias seguidas de mesma letra, na coluna, não diferem estatisticamente entre si pelo teste $\mathrm{t}(\mathrm{P}>0,05)$.

desta espécie, obtendo-se níveis médios de $96,5 \%$ aos 60 DAA; nas doses de 75 e $150 \mathrm{~mL} \mathrm{ha}^{-1}$, ele apresentou pouca eficácia. Já o Roundup (3,0 L ha-1) foi muito pouco eficaz no controle desta planta.

No entanto, quando se aplicou Aurora 400 $\mathrm{CE}+$ Roundup $\left(75 \mathrm{~mL}+3,0 \mathrm{~L} \mathrm{ha}^{-1}\right)$, houve eficácia no controle, proporcionando níveis médios de $80 \%$ aos 60 DAA. Em relação ao Aurora $400 \mathrm{CE}+\operatorname{Arsenal}\left(75 \mathrm{~mL}+2,0 \mathrm{~L} \mathrm{ha}^{-1}\right)$, o controle também foi eficaz, proporcionando niveis médios de $86,3 \%$ aos 60 DAA (Tabela 3).

De modo geral, para esta espécie, o tratamento que proporcionou a melhor eficácia foi o Aurora $400 \mathrm{CE}\left(300 \mathrm{~mL} \mathrm{ha}^{-1}\right)$, porém a mistura Aurora $400 \mathrm{CE}+$ Roundup $(75 \mathrm{~mL}+$ 3,0 L ha-1) também mostrou eficácia sobre esta planta. Apesar de não ter sido necessária ao Aurora $400 \mathrm{CE}\left(300 \mathrm{~mL} \mathrm{ha}^{-1}\right)$ a mistura com Roundup, esse resultado demonstra novamente que a mistura é possivel, já que este último isolado tem pouca eficácia de controle desta espécie.

\section{Salvinia (Salvinia auriculata)}

Pode-se observar, na Tabela 4, que apenas o tratamento Aurora $400 \mathrm{CE}+$ Roundup $(75 \mathrm{~mL}$ $+3,0 \mathrm{~L} \mathrm{ha}^{-1}$ ) foi eficaz no controle desta espécie, proporcionando níveis médios de $82,5 \%$ aos 60 DAA. Em relação aos demais tratamentos, houve pouca eficácia no controle. Apesar de os tratamentos Aurora $400 \mathrm{CE}$ (300 mL ha ${ }^{-1}$ ) e Aurora $400 \mathrm{CE}+$ Arsenal (75 mL 2,0 L ha-1) terem alcançado niveis de controle acima de $80 \%$ aos 20 DAA, ocorreu recuperação das plantas e o controle tornou-se ineficaz até os 60 DAA (Tabela 4).

Para esta espécie, apenas o tratamento 6 (Aurora $400 \mathrm{CE}+$ Roundup com $75 \mathrm{~mL}+$ 3,0 L ha-1) apresentou-se eficaz, o que confirma o bom desempenho da mistura.

Num contexto geral, a mistura de Aurora $400 \mathrm{CE}+$ Roundup $\left(75 \mathrm{~mL}+3,0 \mathrm{~L} \mathrm{ha}^{-1}\right)$ proporcionou a melhor eficácia de controle sobre as plantas de aguapé (E. crassipes), alface-d'água (P. stratiotes) e salvinia (S. auriculata) e, além disso, confirmou-se o bom desempenho da mistura utilizada.

\section{LITERATURA CITADA}

KISSMANN, K. G. Plantas infestantes e nocivas. 2.ed. São Paulo: BASF, 1997. Tomo I. 824 p.

LORENZI, H. Plantas daninhas do Brasil: terrestres, aquáticas parasitas e tóxicas. 3.ed. Nova Odessa-SP: Plantarum, 2000. $608 \mathrm{p}$.

SWARBRICK, J. T. Weeds of Australia: Salviniaceae, Primulaceae, Pontederiaceae. Aust. Weeds, v. 1, n. 10, p. 21-27, 1981.

Planta Daninha, Viçosa-MG, v. 23, n. 2, p. 305-310, 2005 
UNIVERSITY OF FLORIDA. Aquatic, wetland and invasive plant particulars and photographs. Disponível em: $<$ http://aquat1.ifas.ufl.edu/cedepic.html $>$. Acesso em: 04 fev. 2004
VELINI, E. D. Controle mecânico de plantas aquáticas no Brasil. In: WORKSHOP SOBRE CONTROLE DE PLANTAS AQUÁTICAS, 1998, Brasília. Anais... Brasília: IBAMA, 1998. p. 32-35. 